\begin{tabular}{|c|c|c|}
\hline 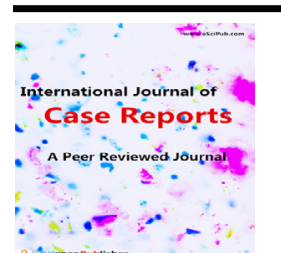 & $\begin{array}{l}\text { International Journal of Case Reports } \\
\text { (ISSN:2572-8776) }\end{array}$ & 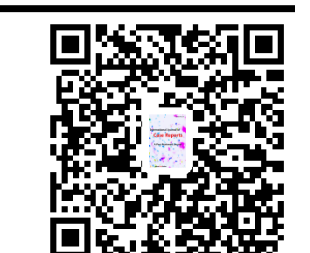 \\
\hline
\end{tabular}

\title{
Advances in Diagnosis of Mitochondrial Diseases: Case Report of an Infant with Pearson Syndrome
}

\section{Lilian Cohen MD, MPH ${ }^{1 *}$ and Ana Maria Rodriguez Barreto MD ${ }^{1^{*}}$, Miguel Dario Cantu MD², Catherine McGuinn MD ${ }^{1}$, Madhu Ouseph MD, PhD $^{2}$, Sanjay Patel MD, MPH${ }^{2}$, Shipra Kaicker MD' \\ ${ }^{1}$ Department of Pediatrics, Weill Cornell Medical College, New York Presbyterian Hospital; ${ }^{2}$ Department of Pathology and Laboratory Medicine, Weill Cornell Medical College, New York Presbyterian Hospital}

ABSTRACT
Pearson syndrome (PS) is a mitochondrial disorder that presents
in early infancy as a multisystemic disease affecting the bone
marrow and pancreas. It may present with anemia, diarrhea,
exocrine pancreatic dysfunction, and failure to thrive. ${ }^{[1]}$ Delay in
diagnosis can lead to severe morbidity and mortality in infan-
cy. We report the case of a 9-month-old presenting with failure
to thrive, severe macrocytic anemia and pancytopenia initially
thought to have gastroesophageal reflux and feeding intolerance.
Severe macrocytic anemia and pancytopenia prompted an early
bone marrow evaluation. Abnormal bone marrow findings includ-
ing vacuolated marrow precursors and ringed sideroblasts along
with persistent mild lactic acidosis led to a rapid and extensive
genetics workup. Whole exome sequencing including mitochon-
drial genome sequencing detected a 2.3 kb heteroplasmic dele-
tion in m.12113_14421 encompassing the MT-ND5 gene consis-
tent with the diagnosis of Pearson Syndrome. This case report
highlights the advances in molecular genetic testing to diagnose
patients with complex medical histories along the spectrum of
mitochondrial diseases and the importance of early diagnosis to
start treatment.
Keywords: Mitochondrial disease, Pearson Syndrome, Molecular Genetic Testing, Whole Exome Sequencing, Mitochondrial DNA.

Abbreviations: Pearson syndrome (PS); mitochondrial DNA (mtDNA); Kearns-Sayre syndrome (KSS); Mitochondrial Encephalomyopathy, lactic acidosis and stroke-like episodes (MeLAS); Hematopoietic Stem Cells for Transplantation (HSCT), Preimplantation Genetic Testing (PGT), Human Leukocyte Antigen (HLA).

*Correspondence to Author:

Ana Maria Rodriguez Barreto MD

Department of Pediatrics-Division of Medical Genetics, Weill Cornell Medical College, 505 East 70th St. 3rd Floor Box 128, New York, New York 10021 USA

How to cite this article:

Lilian Cohen, Ana Maria Rodriguez Barreto, Miguel Dario Cantu, Catherine McGuinn, Madhu Ouseph, Sanjay Patel, Shipra Kaicker. Advances in Diagnosis of Mitochondrial Diseases: Case Report of an Infant with Pearson Syndrome. International Journal of Case Reports, 2021,5:218.

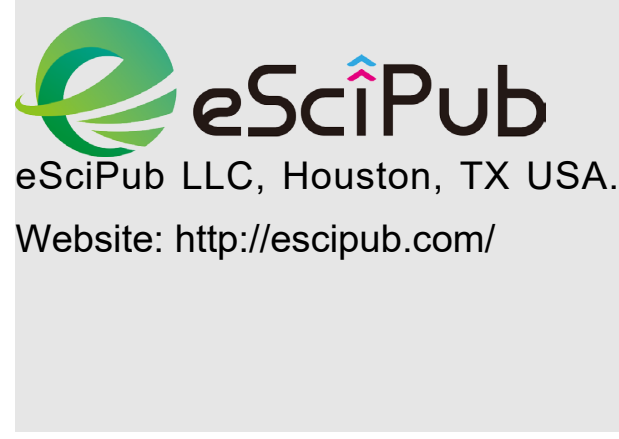




\section{INTRODUCTION}

Pearson syndrome (PS) is a rare constitutional disorder usually caused by single, large-scale mitochondrial DNA deletions that result in bone marrow failure and severe macrocytic sideroblastic anemia. ${ }^{[1-5]}$ It affects multiple organ systems, and is frequently associated with pancreatic insufficiency resulting in steatorrhea and malabsorption. ${ }^{[5]}$ Prognosis is poor ( 20\% 5 -year survival) due to the multifaceted nature of the disease, which includes defective cellular metabolism, malabsorption, renal and hepatic failure, and endocrine dysfunction. We are reporting a 9-month-old male who presented with severe macrocytic anemia and severe pancreatic insufficiency requiring multiple blood transfusions and pancreatic enzyme replacement.

Mitochondrial diseases are complex disorders with varying effects in different organs such as Kearns-Sayre syndrome, progressive external ophthalmoplegia, diabetes, deafness, various ophthalmological and neurological disorders, as well as mitochondrial encephalomyopathy, lactic acidosis and stroke-like episodes (MeLAS). Mitochondria, which contain circular double stranded mtDNA contains 37 genes encoding enzymes involved in the oxidative phosphorylation pathway, is present in all body cells except mature red blood cells. PS is caused by deletions, duplications or pathogenic variants within mitochondrial DNA (mtDNA) that lead to defects in the mitochondrial respiratory chain and defective energy production ${ }^{[5,10,15]}$. Like other mitochondrial diseases, it affects organs with higher energy requirements ${ }^{[6]}$. Mutations are usually sporadic, but germline mutations have also been reported. [10,14,15] Some studies suggest that the severity of disease depends on the size of the deletion and/or level of heteroplasmy. Despite limited case reports in the scientific literature, the true prevalence of PS remains unknown as these mitochondrial syndromes likely remain under diagnosed around the world. Despite supportive treatment, children with PS have a poor prognosis and most die before 3 years of age ${ }^{[8,12]}$. There is limited data for those who survive infancy with features of Kearns-Sayre syndrome (KSS) such as ophthalmoplegia, retinopathy, ataxia and cardiac conduction, hearing and neurological defects ${ }^{[5,8,12]}$. Recent advances in genome sequencing has led to earlier detection by genetic analysis of mtDNA and allow families to access better clinical care and novel treatments.

\section{CASE REPORT}

The patient is a 9-month-old male infant with poor weight gain who presented to a community hospital for vomiting and respiratory distress. Parents reported he had been more tired and paler than usual for the past month. He had poor weight gain for three months prior to this admission and now along $1^{\text {st }}$ percentiles. He had been diagnosed by $\mathrm{Gl}$ with gastroesophageal reflux disease (GERD) and milk protein allergy after multiple episodes of non-bloody, non-bilious emesis. Despite changes in formula, he had worsening symptoms for the last two weeks. He was born at 36 weeks' gestation to a 29-year-old G1P0 mother and 31 year old father of European ancestry. Pregnancy was unremarkable. Birth history significant for premature rupture of membranes. Birth weight $2.863 \mathrm{~kg}$. Family history unremarkable except for a 27-year-old maternal uncle who died suddenly of unknown cause. There had been no major health or developmental concerns though pediatric visits were rather limited while living away with grandparents during COVID-19 pandemic.

In an outside emergency room, he was found to have severe anemia with hemoglobin of $2 \mathrm{~g} / \mathrm{dL}$, neutropenia, and mild thrombocytopenia. He received a red cell transfusion and was transferred to our academic center for hematology workup and further evaluation. Physical exam on admission was significant for weight and height below $1^{\text {st }}$ percentile for age $(7 \mathrm{~kg}$ and $65 \mathrm{~cm}$, respectively) and pale appearance. No congenital anomalies or major dysmorphisms noted. Initial laboratory findings 
showed the following: WBC $4.910 \%$, quantitative PCR was negative. Ceruloplasmin, hemoglobin $2.0 \mathrm{~g} / \mathrm{dL}$, hematocrit 6.2\%, MCV 120 zinc, and copper levels were normal. Fecal $\mathrm{fL}$ and platelets $12410^{9} / \mathrm{L}$. There was a relative reticuloctopenia for the degree of anemia (2.58\%, absolute count $\left.0.65410^{12} / \mathrm{L}\right)$. Lactic acid levels were elevated to $6.10 \mathrm{mmol} / \mathrm{L}$. Vitamin B12, folate, methylmalonic acid and homocysteine levels were normal. Direct Coombs was negative. Plasma parvovirus B19 pancreatic elastase was low $(75 \mu \mathrm{g} / \mathrm{gram})$, suggestive of pancreatic insufficiency. He had intermittent bradycardia and echocardiogram showed mild LV dilation with borderline LVH concerning for potential cardiomyopathy. MRI of brain and ophthalmology exam was unremarkable for age.

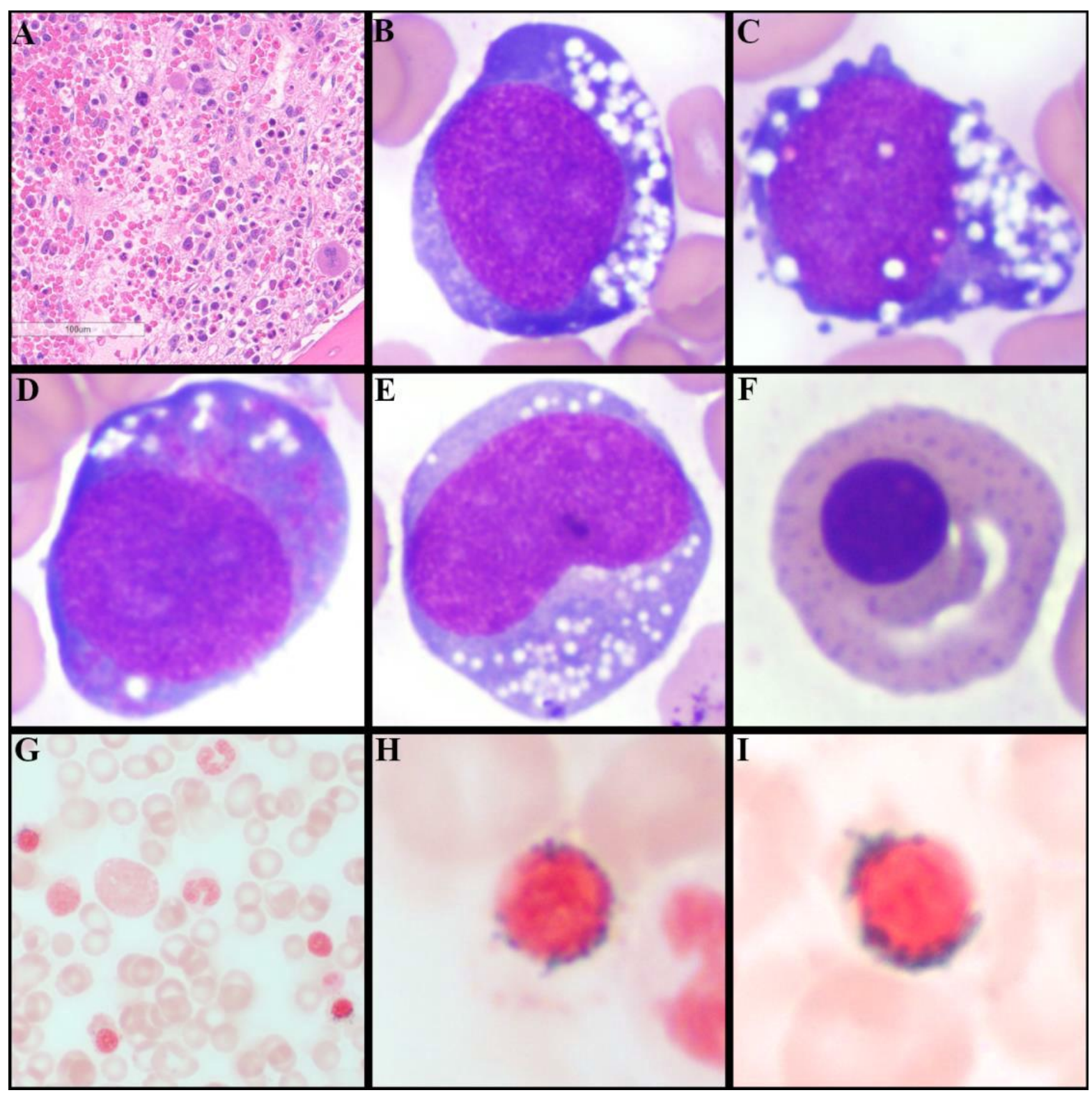

Figure 1: Hematoxylin-eosin stained bone marrow core biopsy shows maturing trilineage hematopoiesis, 200x magnification (panel A). Pronormoblasts with cytoplasmic and occasional nuclear vacuoles, 1000x magnification (panels B-C). Promyelocyte (panel D) and myelocyte (panel E) with prominent cytoplasmic vacuoles, 1000x magnification. Orthochromic normoblast with megaloblastoid features and basophilic stippling, 1000x magnification (panel F). Bone marrow aspirate iron stain with multiple ring sideroblasts and erythroid precursor with few vacuoles, 500x magnification (panel G). Representative ring sideroblasts, 1000x magnification (panels $\mathrm{H}-\mathrm{I}$ ). 
Bone marrow examination was performed on the second day of admission to our institution and identified a hypercellular marrow with maturing trilineage hematopoiesis (Figure 1). Bone marrow aspirate smears showed prominent cytoplasmic and occasional nuclear vacuolization in hematopoietic precursors (B-E), involving both erythroid (B-C) and myeloid lineages (D-E). (Significant basophilic stippling and megaloblastoid features were seen in some nucleated red blood cells (F). An iron stain (Prussian Blue stain) performed on the bone marrow aspirate smear showed approximately $50 \%$ ring sideroblasts $(\mathrm{G}-\mathrm{H})$. An abnormal blast immunophenotype was not detected by flow cytometric analysis ruling out a hematological malignancy. Bone marrow cytogenetics was normal. Differential diagnosis at this point included bone marrow failure syndromes such as Pearson's Syndrome and Diamond Blackfan anemia; copper deficiency, zinc toxicity, myelodysplastic syndromes, inborn errors of folate and cobalamin transport and metabolism and other rare conditions causing sideroblastic anemia with megaloblastic changes. The presence of vacuolated precursors and sideroblastic anemia raised the suspicion of Pearson Syndrome.

Comprehensive genetics work up was expedited to clarify the underlying genetic cause for the patient's complex phenotype. Newborn screen was negative. Preliminary metabolic screening including plasma amino acids, urine organic acid, acylcarnitine studies, ammonia, and $\mathrm{LDH}$, were not suggestive of classic inborn errors of metabolism. Additional molecular studies were obtained with informed consent. Genomic DNA was extracted from peripheral blood samples and testing performed at GeneDx Lab. Chromosomal microarray and whole exome sequencing was expedited but unremarkable for microdeletions, microduplications, or pathogenic variants respectively. The entire mitochondrial genome was sequenced and detected a $2.3 \mathrm{~kb}$ heteroplasmic deletion including m.12113_14421, encompassing the following mitochondrial genes: MT-ND4, MT-TH, MT-TS2, MT-TL2, MT-ND5 and MT-ND6 (heteroplasmy of approximately $81 \%$ ). The deletion was consistent with the diagnosis of Pearson Syndrome.

\section{DISCUSSION}

PS has been previously described as a multisystemic mitochondrial disease presenting with macrocytic anemia, failure to thrive, exocrine pancreatic dysfunction, as well as possible hepatic and renal impairment. Refractory sideroblastic anemia is the most common presentation and is frequently associated with different degrees of thrombocytopenia and neutropenia ${ }^{[1,3,11]}$. A typical finding is striking vacuolization of hematopoietic precursors ${ }^{[8,11,12]}$ which was what raised clinical suspicion for PS in our patient. Low fecal pancreatic elastase can be indicative of pancreatic insufficiency. Exocrine pancreatic dysfunction occurs secondary to fibrosis and is relatively specific for PS ${ }^{[5]}$. However, it is not present in most of the patients early on as prevalence increases with age, ranging from $12.7 \%$ at the time of diagnosis to $18 \%$ at 4 years of age ${ }^{[11]}$. Low birth weight can be more common than exocrine pancreatic dysfunction and presents in $63 \%$ of neonates with PS ${ }^{[12]}$.

In this case study, we successfully confirmed the genetic diagnosis in an infant with PS after extensive series of testing and relatively rapidly allowing us to institute treatment. Upon diagnosis, our patient's pediatric care has been coordinated with specialists from hematology, $\mathrm{Gl}$, genetics, cardiology, ophthalmology, audiology, endocrinology, and development. The family was referred to several mitochondrial advocacy groups, including the CHAMP Foundation for Pearson Syndrome, as well as a mitochondrial disease network of specialists. Our patient was recommended to start enzyme replacement therapy for severe pancreatic insufficiency as well as a mitochondrial cocktail (Citrulline, Ubiquinol, Riboflavin, Vitamin E, Folinic acid, and a Multivitamin) by mitochondrial clinic team at Children's Hospital of Philadelphia. Currently 
he is transfusion dependent and requires PRBC transfusions every 4-6 weeks to maintain a hemoglobin $>8 \mathrm{gram} / \mathrm{dl}$. He is stable with the above management and has had resolution of his feeding intolerance, irritability and is gaining weight and catching up on developmental milestones. He has not had any episodes of infections, sepsis or worsening metabolic acidosis.

Given the genotype-phenotype variability mentioned above, PS remains likely under diagnosed in infants around the world. Without a bone marrow aspirate and genetic testing based on clinical suspicion, the diagnosis may be delayed for an infant with chronic anemia and failure to thrive. Not uncommonly patients with Pearson's syndrome are misdiagnosed with Diamond Blackfan anemia which is a much more common inherited cause of bone marrow failure in infancy. In our case, our patient had a heteroplasmy of approximately $81 \%$. His deletion was $2.3 \mathrm{~kb}$ which is relatively smaller than other published cases of about $5.0 \mathrm{~kb}^{[12]}$ and it is unclear if smaller sizes could contribute to a milder presentation. Lactic acidosis is a concerning finding and should be monitored closely as most of the patients with PS die from metabolic acidosis early in life ${ }^{[11]}$. Our patient had persistently elevated levels of lactic acid of about 5 during his admission but never developed serious metabolic acidosis. As any deletion in the mtDNA gene is expected to cause a defect in the oxidative phosphorylation pathway, it remains unclear how different levels of heteroplasmy in different tissue can lead to variability of clinical manifestations among $P S$ patients at different ages ${ }^{[7]}$.

Treatment for PS to date has been mainly supportive--blood transfusions as required for anemia, pancreatic enzymes replacement, bicarbonate to control metabolic acidosis and GCSF to reverse infections in the case of severe neutropenia ${ }^{[8,12]}$. Hematopoietic stem cell transplant has been considered as an option to manage the hematological morbidities, but prognosis with this modality has been reported to be poor in PS, and it can be associated with severe complications, such as encephalopathy, renal tubular damage and cancer development ${ }^{[7]}$ A case of successful cord blood transplantation for molecular proved Pearson disease with correction in both hematological abnormalities as well as metabolic acidosis and liver abnormalities over a 3-year post transplant follow up was anecdotally reported by Hoyoux et al in Belgium. Another child with Pearson syndrome was recently transplanted using umbilical cord blood stem cells from his fully matched younger brother with a short follow up post-transplant but appears to be doing well (The Champ

Foundation website; unpublished). For our patient, we are discussing IVF with PGD for HLA as an option for conceiving a sibling to donate cord blood or hematopoietic stem cells for transplantation (HSCT) which improves overall survival compared with unrelated or nonmatched donors ${ }^{[17,18,19]}$. PGD for HLA allows genetic testing of IVF embryos to identify those not carrying a given disease causing mutation and, HLA matching, to achieve conception of a healthy child who could be a matched donor for their sibling ${ }^{[17,19]}$.

Advances in molecular testing are becoming increasingly available in the clinical setting leading to earlier diagnosis and treatment. Improvements in sequencing technology, bioinformatics, commercial lab turnaround time for results, and better insurance coverage have allowed more patients to access genetic testing. Our case highlights both whole exome sequencing (WES) and mitochondrial genome sequencing are important to clarify conditions characterized by genetic heterogeneity. As with any genetic test, it is important for families to have appropriate counseling and education about risk, benefits and limitations of genetic testing by medical geneticists and genetic counselors. As molecular genetic testing improves, confirming a genetic etiology in pediatrics can provide valuable information for timely surveillance, intervention, therapy, and 
preventive medical care.

\section{Acknowledgement}

We thank the patient and family for their participation and contribution.

Dr. Lilian Cohen MD MPH and Dr. Ana Maria Rodriguez Barreto MD contributed equally to this case report.

\section{REFERENCES}

[1]. Farruggia P, Di Marco F, Dufour C. Pearson syndrome. Expert Rev Hematol. 2018 Mar;11(3):239-246. doi: 10.1080/17474086.2018.1426454. Epub 2018 Jan 23. PMID: 29337599.

[2]. Rötig A, Cormier V, Blanche S, Bonnefont JP, Ledeist $F$, Romero N, Schmitz J, Rustin $P$, Fischer A, Saudubray JM, et al. Pearson's marrow-pancreas syndrome. A multisystem mitochondrial disorder in infancy. J Clin Invest. 1990 Nov;86(5):1601-8. doi: 10.1172/JCl114881. PMID: 2243133; PMCID: PMC296909.

[3]. Rötig A, Bourgeron T, Chretien D, Rustin P, Munnich A. Spectrum of mitochondrial DNA rearrangements in the Pearson marrowpancreas syndrome. Hum Mol Genet. 1995 Aug;4(8):1327-30. doi: $10.1093 / \mathrm{hmg} / 4.8 .1327$. PMID: 7581370.

[4]. Pearson HA, Lobel JS, Kocoshis SA, Naiman JL, Windmiller J, Lammi AT, Hoffman R, Marsh JC. A new syndrome of refractory sideroblastic anemia with vacuolization of marrow precursors and exocrine pancreatic dysfunction. J Pediatr. 1979 Dec;95(6):976-84. doi: 10.1016/s00223476(79)80286-3. PMID: 501502.

[5]. Wild KT, Goldstein AC, Muraresku C, Ganetzky RD. Broadening the phenotypic spectrum of Pearson syndrome: Five new cases and a review of the literature. Am J Med Genet A. 2020 Feb;182(2):365-373. doi: 10.1002/ajmg.a.61433. Epub 2019 Dec 11. PMID: 31825167; PMCID: PMC7183758.

[6]. Jennifer MS, Cortez D. Pearson marrowpancreas syndrome with cardiac conduction abnormality necessitating prophylactic pacemaker implantation. Ann Noninvasive Electrocardiol. 2020 Jan;25(1):e12681. doi: 10.1111/anec.12681. Epub 2019 Sep 1. PMID: 31475425 ; PMCID: PMC7358881.

[7]. Faraci et al. Allogeneic bone marrow transplantation for Pearson's syndrome. Bone Marrow Transplant 2007; 39:563-565.

[8]. Hoyoux et al. Letters to the Editor, Cord Blood Transplantation with Pearson's disease; Pediatric Blood Cancer 2008;566-569.
[9]. Reddy JM, Jose J, Prakash A, Devi S. Pearson syndrome: a rare inborn error of metabolism with bone marrow morphology providing a clue to diagnosis. Sudan J Paediatr. 2019;19(2):161-164. doi: 10.24911/SJP.106-1534158413. PMID: 31969746; PMCID: PMC6962262.

[10]. Akesson LS, Eggers S, Love CJ, Chong B, Krzesinski El, Brown NJ, Tan TY, Richmond CM, Thorburn DR, Christodoulou J, Hunter MF, Lunke S, Stark Z. Early diagnosis of Pearson syndrome in neonatal intensive care following rapid mitochondrial genome sequencing in tandem with exome sequencing. Eur J Hum Genet. 2019 Dec;27(12):1821-1826. doi: 10.1038/s41431-019-0477-3. Epub 2019 Jul 29. PMID: 31358953; PMCID: PMC6871088.

[11]. Finsterer J, Scorza FA, Scorza CA. Broad Phenotypic Heterogeneity and Multisystem Involvement in Single mtDNA Deletion-associated Pearson Syndrome. Med Arch. 2018 Jun;72(3):234-236. doi: 10.5455/medarh.2018.72.234-236. PMID: 30061775; PMCID: PMC6021152.

[12]. Khasawneh $R$, Alsokhni $H$, Alzghoul B, Momani A, Abualsheikh N, Kamal N, Qatawneh M. A Novel Mitochondrial DNA Deletion in Patient with Pearson Syndrome. Med Arch. 2018 Apr;72(2):148-150. doi: 10.5455/medarh.2018.72.148-150. PMID: 29736106; PMCID: PMC5911175.

[13]. Farruggia $P$, Di Cataldo A, Pinto RM, Palmisani E, Macaluso A, Valvo LL, Cantarini ME, Tornesello A, Corti P, Fioredda F, Varotto S, Martire B, Moroni I, Puccio G, Russo G, Dufour C, Pillon M. Pearson Syndrome: A Retrospective Cohort Study from the Marrow Failure Study Group of A.I.E.O.P. (Associazione Italiana Emato-Oncologia Pediatrica). JIMD Rep. 2016;26:37-43. doi: 10.1007/8904_2015_470. Epub 2015 Aug 4. PMID: 26238250; PMCID: PMC4864774.

[14]. Muraki K, Sakura N, Ueda H, Kihara H, Goto $Y$. Clinical implications of duplicated mtDNA in Pearson syndrome. Am J Med Genet. 2001; 98(3): 205-209.

[15]. Jacobs LJ, Jongbloed RJ, Wijburg FA, de Klerk JB, Geraedts JP, Nijland JG, Scholte HR, de Coo IF, Smeets HJ. Pearson syndrome and the role of deletion dimers and duplications in the mtDNA. J Inherit Metab Dis. 2004; 27(1): 47-55.

[16]. Sato T, Muroya K, Hanakawa J, Iwano R, Asakura Y, Tanaka Y, Murayama K, Ohtake A, Hasegawa T, Adachi M. Clinical manifestations and enzymatic activities of mitochondrial respiratory chain complexes in Pearson marrowpancreas syndrome with 3-methylglutaconic IJCR: https://escipub.com/international-journal-of-case-reports/ 
aciduria: a case report and literature review. Eur J Pediatr. 2015; 174(12): 1593-602.

[17]. Crippa BL, Leon E, Calhoun A, Lowichik A, Pasquali $M$, Longo $N$. Biochemical abnormalities in Pearson syndrome. Am J Med Genet A. 2015; 167A(3): 621-628.

[18]. Tur-Kaspa I, Jeelani R. Clinical guidelines for IVF with PGD for HLA matching. Reprod Biomed Online. 2015 Feb;30(2):115-9. doi: 10.1016/j.rbmo.2014.10.007. Epub 2014 Oct 18. PMID: 25500181.

[19]. Horan, J., Wang, T., Haagenson, M., Spellman, S.R., Dehn, J., Eapen, M., Frangoul, H., Gupta, V., Hale, G.A., Hurley, C.K., Marino, S., Oudshoorn, M., Reddy, V., Shaw, P., Lee, S.J., Woolfrey, A., 2012. Evaluation of HLA matching in unrelated hematopoietic stem cell transplantation for nonmalignant disorders. Blood 120, 2918-2924.

[20]. Kahraman, S., Beyazyurek, C., Yesilipek, M.A., Ozturk, G., Ertem, M., Anak, S., Kansoy, S., Aksoylar, S., Kuskonmaz, B., Oniz, H., Slavin, S., Karakas, Z., Tac, H.A., Gulum, N., Ekmekci, G.C., 2014. Successful hematopoietic stem cell transplantation in 44 children from healthy siblings conceived after preimplantation HLA matching. Reprod. Biomed. Online 29, 340-351. 